Hospital Manuel Arriatán.

Sección Múdicina Interna

Prof. E. Cienfueges.

Policlínica de Lúes.

\title{
ENSAYO DE PIRETOTERAPIA EN DOS ENFERMOS CON QUERATITIS PARENQUIMATOSA POR LUES CONGENITA
}

\author{
Pot e! Dr. ALFREDO DABANCENS 1.
}

La presente comunicación tiene por objeto dar a cono. cer los resultados obtenidos en un ensayo de piretoterapia por baños calientes practicado en 2 niños afectos de queratitis parenquimatosa por lúes congénita.

1.' Observación: Ernesto M., 13 años. N. Reg. 4017. Ingresó el $1.0^{\circ}$-III-43.

Antecedentes familiares. - Padre abandonó el hogar y no se conocen sus antecedentes. Madre cré́a ser sana, pero examinada en el Servicio, dió Wass. $t++, \mathrm{Kahnn}++, \mathrm{Ha}$ tenido 9 hijos, de los cuales sólo viven 3; la hija mayor presenta estigmas de lúes congénita y serología intensamente positiva; el hijo menor es seronegativo. No hay antecedentes de tuberculosis. al año.

Antecedentes personales. - Nació a tétmino. Anduvo

Enfermedades anteriores: Ha sido sano. En julio de 1941 se le hizo Pirquet, el que resultó positivo.

Enfermedad actual. - En enero de 1942 comienza a sufrir de fotofobia, epifora y disminución áe la agudeza visual, por lo que al cabo de una semana consultá al Policlínico de Oftalmología, donde se comprueba inyección ciliar y cónea blanquecina $O$. 1. Serología: Wass. + , Kahn +++ . 
O. I.

Diagnóstico. - Lúes congénita: queratitis intersticial

Tratamiento. - Local con pomada de atropina y general con leche aséptica inyectable. Se envía al Policlínico de I úes para su tratamiento específico.

Evolución. - Cuando llevaba solamente 3 dosis de Neo, el thiño se siente bastante mejor y abandona el tratamiento, permaneciendo inasistente un año entero. En febrero $\mathrm{d}_{\mathrm{t}} 1943$ vuelve a consultar por inyección conjuntival, epífora y fotofobia, esta vez del ojo derecho. Se diagnostica una nueva queratitis parenquimatosa y se reanuda el tratamiento especifico con Neo en forma intensiva. o sea inyecciones día por medio en dosis de 1.6 centigramo por $\mathrm{kgr}$. de peso y llega así a enterar. 6 grs. de Neo en 35 días, lo que da una dosis total por $\mathrm{kgr}$. de 16 centígramos. A pisar de esta arsenoterapia intensiva, sus lesiones oculares, lejos de mejorar, tienden más bien a intensificarse y se bilateralizan como lo indican las siguientes anotaciones del oculisea:

3 de marzc:" "Ha aumentado la inyección ciliar fotofobia y epífora $O$. D. I.

18 de marzo: $O$. D. I. Córneas infiltradas. Intensa inyección ciliar y pupilas no dilatan".

En estas circunstancias proponemos a la Dra. Candia (cculista) ensayar la piretoterapia, mediante baños calientes. la que es aceptada por ella e iniciada a cargo del suscrito el día 2 de abril de 1943, con la técnica que se describirá más adelante y después de practicar los exámenes de rigor, a saber: Radiosccpia; examen de orina y presión arterial, todos los cua'es resultaron normales.

Se practicaron en total 5 baños calientes, en las fechas $2,5,8,15$ y 20 de abril, con buena tolerancia de parte del crfermo, quien, en un comienzo se resistía a mantenerse en il baño durante el tiempo requeridc por sentirse sofocado y fatigado, pero ya después del $2 .^{\circ}$ baño. los aceptó de mejor grado, porque notó una apreciable mejoría de su vista. En efecto, la fotofcbia y epífora, que son los síntomas subjetivos más mortificantes de la queratitis parenquimatosa, fueron disminuyendo hasta desaparecer totalmente $y$, también desde el punto de vista objetivo se pudo apreciar como las cómeas iban limpiàndose lentamente $\mathrm{y}$ dismiruyendo también ta inyección ciliar.

En los intervalos entre un baño y otro, se colocó cianuro de mercurio al $1 \%$, por vía endovenosa, con un total 
de 9 cc. La marcha francamente regresiva de las lesiones cculares prosiguió aún después de terminada la serie de 5 baños calientés $y$ en ausencia de tratamiento esperifico activo, pues éste se confirmó sólo con jarabe de Gibert.

La serología también fué virando lentamente después de la piretoterapia, al terminar ésta, el Kahn era positivo ++ . al cabo de un mes $+y$ al mes después fué negativo.

Actualmente, el enfermo no tiene molestias subjetivas. ha subido $1 \mathrm{kgr}$. de peso y sólo presenta disminución de la agúdeza vísual por las opacidades residuales de sus córneas, especialmente en $O$. I.

2." Observación. - Luis C.. 10 años. N.9 Reg. 53261. Ingresó el 19-X-42.

Antecedentes familiares. - Padre: 54. años, luético desde hace 18 años. Setopositivo. No se ha tratado. Madre: 37 años, serepositiva. No so ha tratado. Ha ténido 8 hiios. de los cuales 2 han fallecido antes de los 2 años. No hay abortos. No hay antecedentes de tuberculosis.

Antecedentes nersonales. - Nació a término en parto rormat. Alimentado a pecho más de un año, anduvo a los 12 meses.

Enfermedades antericrs:- Sarampión a los 14 meses.

Enfermedad acturl. - Hace alrededor de un mes aue. rresenta irritación a la vista con epífora y fotofobia progresiva. por lo que consulta al Policlínico de Oftalmologia. donde so comprueba intensa inyección conjuntival y ciliar 0 D. I. Córneas totalmente infiltradas, Fotofobia marcadia. Serclogía Wars.: H. R. Kahn ++ . tosa.

Diagnóstico. - Lúes congénita; quèratitis parenquima-

Tratamiento. - Lccal con pomada de dionina y atropina y específicc con Neoarsolán. Después de una semana de asistir al Políclínico se hospitaliza en Medicina Interna.

Evelución. - Después de 3 meses de tratamiento escecifico con bismuto. fricciones mercuriales y Neoatsolán y lecal con tópicos adecuados, la sintomatología ocular permanece invariable, por lo aue el especialista comienza una serie de inyecciones sub-conjuntivales de suero fisiológico día por medio, hasta enterar unas 6 en total. Posteriormente le indica una cura con extracto de bazo desalbuminizado débil empezando por $2 / 10 \mathrm{cc}$. intradérmico. para seguir con inyecciones subcutáneas progresivas, hasta 1 cc. en númeto de 10 . En seguida se hace una segunda serie de inyecriones subconjuntivales alternadas con inyecciones de extracto de bazo 
fuerte en númerc de 5 cada una. Durante todo este tiempo se ha hecho también tratamiento específico y, además, algunas aplicaciónes de radioterapia superficial, pero el estado ocular no muestra variaciones apreciables y la serología s? mantiene irreductible.

A. esta altura de la evolución, o sea, después de 7 meses de hospitalización fuí llamado en consulta por los colegas del Servicio de Medicina y entonces tuve oportunidad de propener la intensificación del tratamiento específico y la piretoterapia por baño caliente. Con el asentimiento de ls colega oculista, Dra. Candia y previos los exámenes de orina; radicscopía de tórax y presiones arteriales que resultaron normales, el día 18 de marzo de 1943 se practicó el primer bañc caliente. Se hizo en total 5 baños calientes, en las $f e$ chas $18,20,22,24$ y 28 de mayo.

En los intervalos se inyectó Nearsolán en dosis de 1,7 For kilogramo, pero dos de ios baños (el $3 .^{\circ}$ y el $4 .^{\circ}$ ) sé Fracticaron el mismo día que la invección de ngoarsolán unas 3 horas después de ésta. La aceptación por parte del niño no fué de muy buen grado. pues en todos los baños se debatió vivamente por salirse del agua.

De todos mcdos, la enterma logró llenar su difícil cometido y pudo verse la acción favorable del tratamiento por la desaparición de la epífora y de la fotofobia al cabo del tercer baño.

En cuanto a la infiltración corneal, ésta ha sido bastEnte pertinaz: pero indudablemente que ha ido disminuyendo con gran lentitud. En efecto, al cabo de dos meses de terminada la serie de piretoterapia y estando en descanso del tratamiento específico, el niño presenta una opacidad corneal en grado de nubécula en el ojo izquierdo y de leucoma central en el oje derecho. No hay inyección ciliar; no hay epífora ni fotofobia. La seróogía se mantiene igual: Kabn ++ . Fil estado genetal es bastante bueno: ha subidc $1 \frac{1}{2}$ kilo $\mathrm{cm}$ dos meses.

Nuestra intención fué de continuar y repetir la piretoterapia en este niño para alcanzar sta efecto máximo. pero, a pesar de todos nuestros esfuerzos y tentativas, nos fué im?posible hacerlo per una serie de circunstancias materiales adyersas que no hemos podido subsanar. 
Indicaciorres de la piretocerapia en la lúes:

1.-Queratitis parenquimatosa.

2.- Lesiones terciarias de la piel.

3.-Sífilis ósea y articular.

4.-Sífilis hepática.

5. ¿ ¿Neurosífilis?

Contraindicaciones de la piretoterapia:

1.-Tuberculosis activa.

2.-Cardiopatias:

3.-Nefrcpatías.

4.-Enfermedades neurológicas con pérdida de la sensib:lidad.

5.-Caquexia.

6.-Estados demenciales.

7.- ¿Neurosifilis?

Técnica de la piretoterapia con baño caliente (Dennie y Pakula).

Perroniall y útiles necesarios:

a) Una enfermera inteligente (que no le tema al trabajo).

b) Una tina de baño con abundante aguai caliente.

c) Un termómetro para baño.

d) Un termómetro clínico.

Procedimiento:

a) Verificar que no existan contraindicaciones. El enfermo debe someterse a un tiguroso examen físico, incluyendo radioscopía de tórax. comprobación de la presión arterial y examen de orina.

b) El enfermo se coloca dentro de baño en posición sentado: la temperatura del agua debe ser do $36 \mathrm{r} / 2$ a $37 \mathrm{gra}$ dos; el agua debe llegar sólo hasta los mamelones, deiando la axila completamente seca.

c) Para asegurarse de que el enfermo no tenga intolerancia por la piretoterapia se hace ur prímer baño de prueba, en que no se le somete al máximo de calor. Si el enfermo no presenta reacciones anormales, se continúa la serie de baños; en caso contrario, se suspende. 
Primer baño (de prueba): La temperatura del agua se eleva hasta 42 grados en el curso de diez a quince minutos y se mantiene así hasta que la temperatura del niño llegue a $39 \mathrm{~T} / 2$ grados y se mantenga durante diez minutos.

Segundo baño y siguientes: Temperatura inicial del agua: 37.7 a 38 grados. La temperatura del agua se eleva a $44 \frac{1}{2}$ grados en diez a quince minutos y se mantiene ahí rasta que la temperatura del niño suba a 40 ó 41 grados $y$ se mantenga durante 20 minutos.

L.a temneratura dal niño puede medirse en la axila n en la boca. Fl promedio de tiempo necesario nara plevar $t_{7}$ temperatura de! niño desde el comienzo del baño hasta alcanzar 41 grados, es de 35-45 minutos. Como hay que mantenerlo a esta alta temperatura durante 20 minutos, todo el procedimiento ocupa entre una hora a una hora y media.

Una vez enterado el tiempo indicado, el niño es retirado del baño envuelto en frazadas y se acuesta conveniente: mente abrigado.

Sobreviene durante el baño y después de él una copiosa transpiración, que proxoca una sed bastante intensa, por deshidratación. Se debe suministrar líquidos abundantes, de preferencia tibios y azucarados.

La temperatura y el pulso del enfermo se normalizan rápidamente, por lo general en un plazo máximo de tres horas, al cabo de las cuales é enfermo puede levantarse $y$ hacer su vida habitual. 